\title{
Effects of Intravitreal Aflibercept in Patients with Central Serous Chorioretinopathy
}

\author{
Muhammad Afzal Bodla ${ }^{1}$, Ali Afzal Bodla ${ }^{2}$, Syeda Minahil Kazmi ${ }^{3}$, Ayema Moazzam $^{4}$ \\ ${ }^{1-4}$ Department of Ophthalmology, Multan Medical and Dental College, Multan
}

\begin{abstract}
Purpose: To assess the efficacy of intravitreal injection of Aflibercept in patients with chronic central serous chorioretinopathy (CSCR).
\end{abstract}

Study Design: Interventional case series.

Place and Duration of Study: Multan Medical and Dental Centre and Bodla Eye Care from February 2019 to February 2020.

Methods: This study was conducted on fifteen eyes with sub-acute to chronic central serous chorioretinopathy. All patients who had undergone previous treatment with laser photocoagulation, intravitreal triamcinolone, or bevacizumab in past 3 months, history of glaucoma (intraocular pressure $>21 \mathrm{~mm} \mathrm{Hg}$ ), patients who were lost to follow-up, and those with previously vitrectomized eyes were excluded. Patients were treated with a single dose of intravitreal injection of Aflibercept $(0.5 \mathrm{mg} / 0.05 \mathrm{ml})$. Visual acuity with Log MAR chart and central retinal thickness were studied before and after the injection.

Results: The mean age of the patients was 30.46 years \pm 9 years. After one month of intravitreal Aflibercept injection, visual acuity improved from 0.32 Log MAR to 0.173 log MAR and at 3 months (the last follow-up) it was 0.206 Log MAR. Central retinal thickness (CRT) improved from $437 \pm 82 \mu \mathrm{m}$ (at the time of presentation) to $349 \pm$ $67 \mu \mathrm{m}$ at 1 month post injection. At the last follow-up the mean CRT decreased to $309 \pm 121 \mu \mathrm{m}$. Except for two eyes, all eyes showed visual improvement. These two eyes had a higher CRT as compared to other participants.

Conclusion: Intravitreal injection of Aflibercept is effective in improving BCVA and decreasing the central retinal thickness in patients central serous chorioretinopathy.

Key Words: Central retinal thickness, Central Serous chorioretinopathy, Aflibercept.

How to Cite this Article: Bodla MA, Bodla AA, Kazmi SM, Moazzam A. Effects of Intravitreal Injection of Afibercept in Patients with Central Serous Chorioretinopathy. Pak J Ophthalmol. 2020; 36 (4): 402-407.

Doi: https://doi.org/10.36351/pjo.v36i4.1098

\section{INTRODUCTION}

Central serous chorioretinopathy (CSCR) is a chorioretinal disorder distinguished by serous retinal detachments repeatedly engaging the macula and with

Correspondence: Ali Afzal Bodla

Department of Ophthalmology, Multan Medical and Dental College, Multan

Email: aliafzal111@gmail.com

Received: July 9, 2020

Accepted: September 2, 2020 occasional focal pigment epithelial detachments (PED), choroidal hyper-permeability and increased choroidal thickness. ${ }^{1}$ The pathogenesis of central serous chorioretinopathy (CSCR) is inadequately understood and can be associated with systemic involvement, an aetiology influenced by multiple factors and an intricate pathogenesis. Variations from the norm in the choroidal circulation have been conjectured to be causative variables. A few recent findings in CSCR were noted using advanced indocyanine green angiography. These changes include hyper permeability of the choroidal circulation surrounding active retinal pigment epithelial leaks 
which can be extra central and multifocal and numerous assumed serous retinal epithelial separations. $^{2-3}$ Other studies demonstrated an extremely thick choroid in patients with focal serous chorioretinopathy using enhanced depth imaging spectral domain optical coherence tomography. This finding implicates that focal serous chorioretinopathy might be brought about by expanded hydrostatic pressure in the choroid. ${ }^{4}$

Despite the fact that most instances of CSCR are self-limiting, chronicity in few can lead to structural changes of retinal pigment epithelium and a subsequent visual compromise. Other researches show that anti-vascular endothelial growth factor (antiVEGF) therapy can result in decrease in sub retinal fluid in CSCR by lowering choroidal vascular hyper permeability, due to its anti-permeability properties. ${ }^{5-6}$

Aflibercept is a unique and a recombinant protein that consists of parts of vascular endothelial growth factor (VEGF) receptor R1 and (VEGF) receptor R2 with extracellular domains joined to the FC portion of human immunoglobulin G1. ${ }^{7}$ Intravitreally injected Aflibercept is more effective in reducing subfoveal CMT in comparison to Ranibizumab. After Aflibercept injection, choroidal thinning extends beyond the macula. ${ }^{8}$ Intravitreal Aflibercept can accomplish abatement of exudative retinal changes in eyes with wet age related macular degeneration having choroidal neovascularization refractory to Ranibizumab and Bevacizumab. Moreover, it demonstrated more noteworthy outcome in the choroidal and retinal pigment epithelial detachments than the mentioned drugs. ${ }^{9}$ In patients not responding to Ranibizumab and Bevacizumab, Aflibercept has proven to be effective resulting in improvement of CMT. In such patients it can be used as an alternative therapy. In light of these outcomes, Aflibercept's primary power in decreasing leakage from hyper permeable choroidal vessels could be put to use for the treatment of CSCR. ${ }^{10}$

This case series was conducted to highlight the effects of Aflibercept in reducing the CMT and improving the visual acuity in patients with CSCR.

\section{METHODS}

This case series included 15 eyes of patients with semi chronic CSCR (symptoms persisting for 3-4 months). The study included patients aged between 22 years and 40 years. The study included both males and females out of whom majority were males (73.3\%). All patients were from rural and urban population of Southern Punjab, Pakistan. Patients with documented non resolving neurosensory detachment on optical coherence tomography (OCT), active leakage on fluorescein angiography (FA) and with no signs of choroidal neovascularization were included in the study.

All patients who had undergone previous treatment with laser photocoagulation, intravitreal triamcinolone, or bevacizumab in past 3 months, history of glaucoma (intraocular pressure $>21 \mathrm{~mm}$ $\mathrm{Hg}$ ), patients who were lost to follow-up, and those with previously vitrectomized eyes were excluded.

The procedure was done in a single centre, conducted by a single surgeon. A 1cc Insulin syringe was used to inject Aflibercept. It was injected $3.5 \mathrm{~mm}$ from limbus in phakic patients and $3 \mathrm{~mm}$ from limbus in pseudophakics. Post intravitreal injection, a single drop of Ofloxacin with $5 \%$ povidone-iodine was instilled. Patients were checked for retinal artery patency and were prescribed topical Ofloxacin 4 times daily for 5 days.

All eyes were injected with a single dose of intravitreal Aflibercept $(0.5 \mathrm{mg} / 0.05 \mathrm{ml})$. On the next follow-up visit, patients were subjected to best corrected visual acuity (BCVA) on Log MAR chart, intra ocular pressure evaluation using Goldmann tonometer, slit lamp examination and dilated fundus examination. Above mentioned assessments along with an OCT of macula on Angio Vue by Opto Vue was performed at 4 and subsequently 12 weeks. Central retinal thickness as well as change in foveal architecture was assessed on OCT.

Out of 15 eyes included in the study 13 eyes had a complete or near complete resolution of neurosensory retinal elevation by the end of 12 weeks. The two remaining eyes were found to be completely nonresponsive to Aflibercept. Out of these two eyes, one continued to have same central retinal thickness while other had an increase in retinal elevation and CRT at the end of 12 weeks compared to the time of enrolment in the study.

Statistical analysis was done using SPSS version 23. Statistical significance was calculated using the $t$ test. 
Table 1: Baseline Demographic and Clinical Characteristics of the Patients. Best-Corrected Visual Acuity (BCVA) Changes from the Time of Administration of the Injection and during the Follow-up Time.

\begin{tabular}{|c|c|c|c|c|c|c|c|c|c|}
\hline $\begin{array}{l}\text { Case } \\
\text { No. }\end{array}$ & Age & Gender & Eye & $\begin{array}{c}\text { Axial } \\
\text { Length }\end{array}$ & Lens Status & IOP & $\begin{array}{c}\text { At } \\
\text { Presentation } \\
\end{array}$ & $\begin{array}{c}\text { BCVA } \\
1 \text { Month Post- } \\
\text { Injection } \\
\end{array}$ & $\begin{array}{c}3 \text { Months Post- } \\
\text { Injection }\end{array}$ \\
\hline 1. & 30 & $\mathrm{M}$ & OD & $24.5 \mathrm{~mm}$ & Phakic & 20 & 0.0 & 0.0 & 0.0 \\
\hline 2. & 25 & M & OS & $22.9 \mathrm{~mm}$ & Phakic & 18 & 0.2 & 0.0 & 0.0 \\
\hline 3. & 32 & M & OD & $23.3 \mathrm{~mm}$ & Pseudo Phakic & 12.6 & 0.3 & 0.0 & 0.0 \\
\hline 4. & 35 & M & OD & $22.2 \mathrm{~mm}$ & Phakic & 15.4 & 0.5 & 0.1 & 0.1 \\
\hline 5. & 27 & M & OS & $24.7 \mathrm{~mm}$ & Phakic & 16 & 0.1 & 0.0 & 0.1 \\
\hline 6. & 33 & $\mathrm{~F}$ & OS & $22.3 \mathrm{~mm}$ & Phakic & 14.2 & 0.7 & 0.4 & 0.4 \\
\hline 7. & 39 & M & OS & $23.4 \mathrm{~mm}$ & Pseudo Phakic & 12.9 & 0.5 & 0.5 & 0.9 \\
\hline 8. & 22 & M & OS & $23.0 \mathrm{~mm}$ & Phakic & 19.5 & 0.5 & 0.1 & 0.1 \\
\hline 9. & 26 & $\mathrm{~F}$ & OD & $23.1 \mathrm{~mm}$ & Phakic & 16.1 & 0.3 & 0.2 & 0.2 \\
\hline 10. & 37 & M & OS & $23.4 \mathrm{~mm}$ & Phakic & 13.9 & 0.4 & 0.1 & 0.1 \\
\hline 11. & 31 & $\mathrm{~F}$ & OS & $23.5 \mathrm{~mm}$ & Pseudo Phakic & 13.5 & 0.0 & 0.0 & 0.1 \\
\hline 12. & 40 & M & OD & $22.3 \mathrm{~mm}$ & Phakic & 15 & 0.6 & 0.7 & 0.9 \\
\hline 13. & 29 & M & OD & $21.3 \mathrm{~mm}$ & Phakic & 14.4 & 0.2 & 0.0 & 0.0 \\
\hline 14. & 23 & F & OS & $25.9 \mathrm{~mm}$ & Pseudo Phakic & 13.2 & 0.2 & 0.0 & 0.0 \\
\hline 15. & 28 & $\mathrm{M}$ & OS & $23.2 \mathrm{~mm}$ & Phakic & 22.5 & 0.3 & 0.3 & 0.2 \\
\hline
\end{tabular}

\section{RESULTS}

The study included fifteen eyes of fifteen patients (Table 1 ). The mean age was 30.46 years \pm 9 years. The follow-up time was 3 months.

The mean baseline visual acuity at the time of presentation was $0.32 \log$ MAR, improving to 0.173 Log MAR at 1 month and a final visual outcome of 0.205 Log MAR at 3 months. Except two eyes (13.3 $\%)$, rest had visual improvement. This particular group did not respond to the use of Aflibercept. For the rest of the cases $(86.6 \%)$, there was a considerable decrease in CRT at the end of first month, eleven eyes $(73.3 \%)$ continued with persistent improvement of the visual parameters till the end of study i.e. 3 months. There was a considerable decrease in CRT at the end of study, which was relevant with the amelioration in both of BCVA and FA leakage (Graph 1).

By formulating a null hypothesis that Aflibercept is not effective for CSCR, we used the $t$ table to calculate our $\mathrm{p}$ values. Our value post 1 month was 0.02 and 0.03 at 3 months i.e. end of study. Thus, we rejected the null hypothesis.

The mean CRT for all patients at the time of presentation was $437 \pm 82 \mu \mathrm{m}$ which decreased to 349 $\pm 67 \mu \mathrm{m}$ at 1 month (Graph 2). At the last follow-up the mean CRT decreased to $309 \pm 121 \mu \mathrm{m}$.

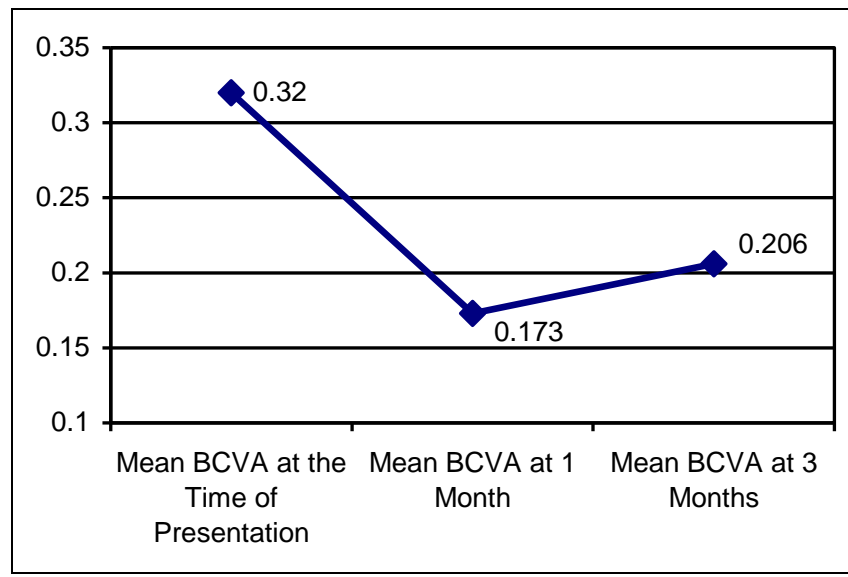

Graph 1: Variations among the mean BCVA at the time of injection, at 1 month and at 3 months post injection administration.

Two of our cases were non-responsive to the use of Aflibercept (Fig. 1). These two cases (Case 7 \& 12) were found to have a higher CRT as compared to other participants. We also observed that the base line $\mathrm{BCVA}$ of non-responding cases were lower than rest of the group. They were found to have a significant level of IS/OS junction breakdown and all these changes in author's opinion led to the poor visual outcome. 


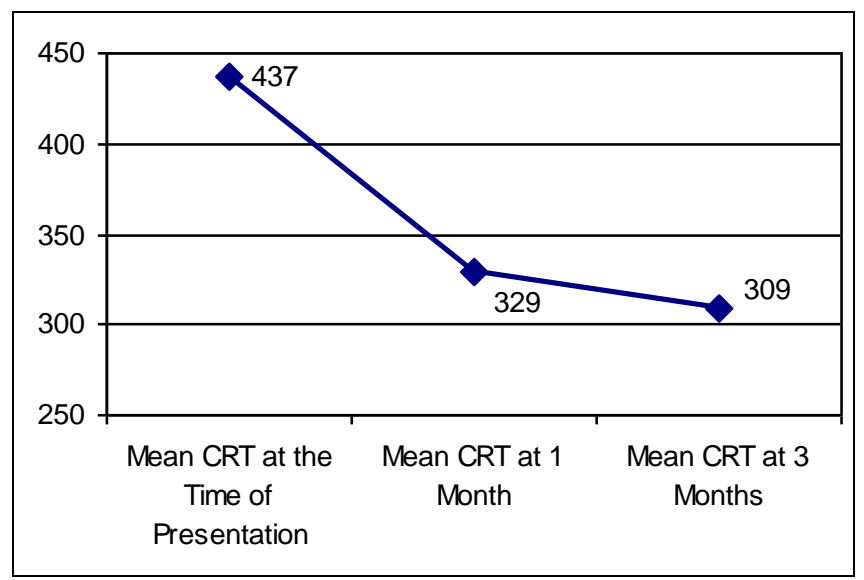

Graph 2: Mean central retinal thickness changes from the start of the treatment and after 1 and 3 months.
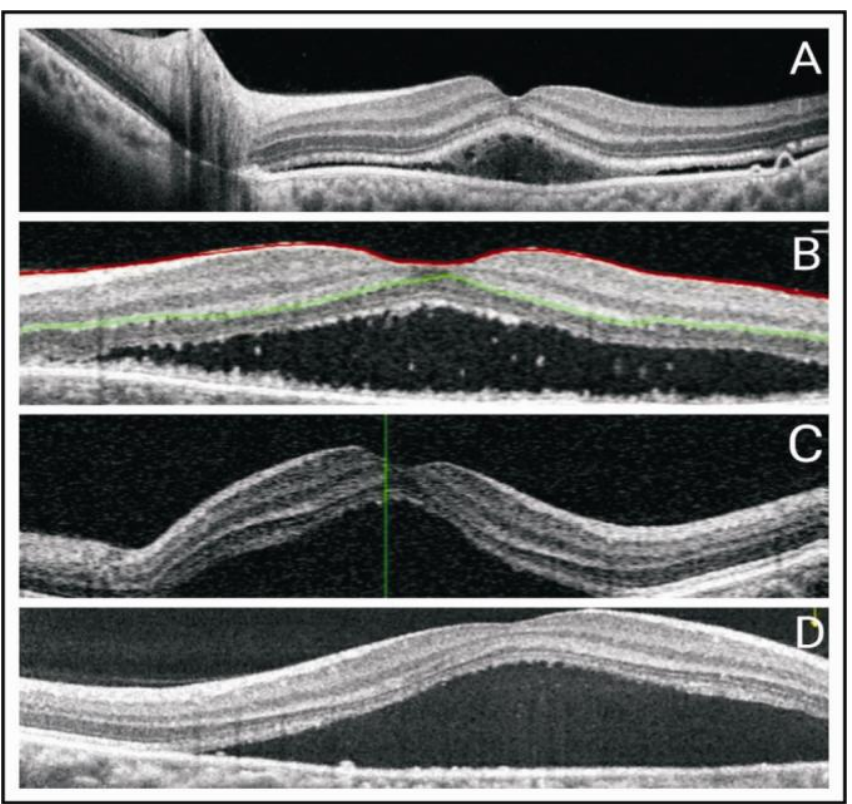

Fig. 1: Spectral domain OCT images of eyes with active CSCR, Fig. A-D. There is presence of a variable amount of sub retinal fluid in the subfoveal region of all patients with an age range of 22 to 40 years. Fig. A (patient 3 ) has minimal SRF and a resolution of symptoms and fluid while in Fig. B (patient 15), Fig. C (patient 6), Fig. D (patient 12), we can appreciate disruption of ISOS layer and presence of debris in sub retinal fluid.

\section{DISCUSSION}

CSCR is a disease characterized by serous separation of the neurosensory retina secondary to one or more focal or diffuse leakage points. Conservative management is preferred by majority of ophthalmologists as a first line option. The actual benefit of immediate resolution may be intervened by a lower rate of RPE degeneration in the concerned eye. ${ }^{11-12}$ The process by which intravitreal bevacizumab therapy improves RPE leak and resorption of sub retinal fluid in CSCR is obscure. Indocyanine green angiography in patients with CSCR has demonstrated signs of choroidal venous congestion and choroidal lobular ischemia. ${ }^{13-15}$ Indicating a generalized RPE or choroidal vascular disturbance. ${ }^{16-18}$ It is a known fact that increased amount of VEGF is released by choroidal ischemia. Only a decrease in VEGF concentration can bring about a positive change in patients with CSCR by ending the series of events, which result in neurosensory detachment. ${ }^{19}$

Several Anti-VEGF agents have been used in the treatment of CSCR like bevacizumab, ranibizumab and Aflibercept. Aflibercept is preferred as it not only attaches multiple isoforms of VEGF-A but also inhibits the activation of VEGFR1and VEGFR2. Aflibercept binds to VEGF-A faster and with higher affinity than bevacizumab and ranibizumab. ${ }^{20}$

As anticipated by a scientific model, Aflibercept has a powerful intravitreal VEGF-binding activity for $10-12$ wk after a single injection. ${ }^{21}$ The biological activity of a therapeutic macromolecule varies on its binding affinity and intraocular half-life. The binding affinity for VEGF of Aflibercept is about 100 times higher than that of ranibizumab or bevacizumab. ${ }^{22}$ The half-life of Aflibercept (molecular weight: $115 \mathrm{kDa}$ ) has not yet been examined in human eyes. As the intraocular half-life of a macro molecule is decided by its molecular size, Aflibercept is likely to have a halflife between ranibizumab (molecular weight: $48 \mathrm{kDa}$ ) and bevacizumab (molecular weight: $149 \mathrm{kDa}) .^{23}$ Hence, the fundamental purpose behind the longer activity of Aflibercept could be clarified by a large increase in binding affinity in comparison to similar elimination half-times.

In our research we suggest that an intravitreal Aflibercept injection in patients with CSCR can induce immediate resorption of sub retinal fluid in majority of cases, and as a result of which there can be a significant improvement in vision. This outcome gave a solid ground for the research and encourage additional investigation of the treatment. ${ }^{19}$

This study showed effective management of CSCR with Aflibercept and none of the patients faced any adverse side effects. Patients with CSCR can undergo natural resolution of symptoms and improvement of retinal architecture. In cases of unilateral CSCR the contralateral eye needs to be 
examined at regular intervals along as there is a probability of subclinical CSCR or development of CSCR in the future. ${ }^{20}$ Further investigations should be carried out to find the role of VEGF in the pathogenesis and treatment of CSCR with anti VEGF agents. ${ }^{19}$

In our study out of improved cases, eleven continued to maintain the improvement in BCVA while two had a subsequent drop in their vision at their third and last visit. Among these two, one eye (case 5) dropped back to the base line BCVA. In this particular case, there was some recurrence of fluid but amount of residual fluid at completion of study was found to be less than what was noticed at the time of presentation. Our other case, (Case 11) had an initial resolution but later the CRT was found to be more than from the time of presentation, which got translated in drop of BCVA at the end of study compared from baseline.

In a previous study Kim et al studied the efficiency of intravitreal ranibizumab injection for acute CSCR in 20 eyes. $^{24} \mathrm{~A}$ dose of $(0.5 \mathrm{mg} / 0.05 \mathrm{ml})$ was given. All patients had increased BCVA, decreased CRT, and resolution of the neurosensory detachment. The overall mean of BCVA in this study improved from $0.77 \mathrm{Log}$ Mar at the start to $0.17 \mathrm{Log}$ Mar after 1 month and stayed static for 6 months. All eyes had visual improvement and eighty percent of patients improved. The results are comparable to our study. However, in our study seventy-four percent of the patients improved.

In another study, Jung et al analysed the response of Aflibercept in acute CSCR. ${ }^{25}$ In their control group there was a significant decrease of retinal thickness and improvement in BCVA. Authors believe that we have seen similar effects in our series of sub-acute and chronic cases, hence pharmacological efficacy of Aflibercept can be of good use in such group of patients. Comparing the rebounding tendency of the disease process, their $11.4 \%$ patients compared to our $15 \%$ had an increase of CRT between 2 and 3 months.

Our study does carry some limitations starting from its relatively small number of eyes without any control group. In our study a single dose of Aflibercept was administered which obviously cannot be compared with the standard practiced regimen of the loading dose of three injections of ranibizumab, bevacizumab or Aflibercept. It would have been interesting to see the effect of three injections of Aflibercept especially for the patients who did not perform well. The reason for minimalistic intervention was the cost limitation in Southern Punjab due to a higher prevalence of a lower socio economic class.

\section{CONCLUSION}

In conclusion, based on acquired data our study supports the efficacy of Aflibercept in terms of improvement in BCVA and reduction of CRT in patients with sub-acute and chronic presentation refractory to self-resolution.

\section{Ethical Approval}

The study was approved by the Institutional review board/Ethical review board.

\section{Conflict of Interest}

Authors declared no conflict of interest.

\section{REFERENCES}

1. Daruich A, Matet A, Marchionno L, Azevedo JD, Ambresin A, Mantel I, et al. Acute Central Chorioretinopathy: Factors Influencing Episode Duration. Retina, 2017; 37 (10): 1905-1915.

2. Guyer DR, Yannuzzi LA, Slakter JS, Sorenson JA, Ho A, Orlock D. Digital indocyanine green video angiography of central serous chorioretinopathy. Arch Ophthalmol. 1994; 112 (8): 1057-1062.

3. Gemenetzi M, Salvo GD, Lotery AJ. Central Serous Chorioretinopathy: An Update on Pathogenesis and Treatment. Eye (Lond). 2010; 24 (12): 1743-1756.

4. Imamura Y, Fujiwara T, Margolis R, Spaide RF. Enhanced depth imaging optical coherence tomography of the choroid in central chorioretinopathy. Retina, 2009; 29 (10): 1469-1473.

5. Inoue M, Kadonosono K, Wantanabe Y, Kobayashi S, Yamane S, Arakawa A. Results of one-year followup examinations after intravitreal bevacizumab administration for chronic central serous chorioretinopathy. Ophthalmologica, 2011; 225 (1): 37 40.

6. Artunay O, Yuzbasioglu E, Rasier R, Sengul A, Bahcecioglu H. Intravitreal bevacizumab in treatment of idiopathic persistant central serous chorioretinopathy: a prospective, controlled clinical study. Curr Eye Res. 2010; 35 (2): 91-98.

7. Trichonas G, Kaiser PK. Aflibercept for the Treatment of Age-Related Macular Degeneration. Ophthalmol Ther. 2013; 2 (2): 89-98. 
8. Yun C, Oh J, Ahn J, Young-Hwang S, Lee B, Woo KS, et al. Comparison of Intravitreal Aflibercept and Ranibizumab Injections on Subfoveal and Peripapillary Choroidal Thickness in Eyes With Neovascular AgeRelated Macular Degeneration. Graefes Arch Clin Exp Ophthalmol. 2016; 254 (9): 1693-1702.

9. Hata M, Oishi A, Tsujikawa A, Yamashiro K, Miyake M, Ooto S, et al. Efficacy of intravitreal injection of Aflibercept in neovascular age-related macular degeneration with or without choroidal vascular hyperpermeability. Invest Ophthalmol Vis Sci. 2014; 55 (12): 7874-7880.

10. Maksys S, Richter-Müksch S, Weingessel B, VẻcseiMarlovits PV. Short-term Effect of Aflibercept on Visual Acuity and Central Macular Thickness in Patients Not Responding to Ranibizumab and Bevacizumab. Wien Kim Wochenschr. 2017; 129 (910): $351-357$.

11. Wang M, Sander B, Larsen M. Retinal atrophy in idiopathic central serous chorioretinopathy. Am J Ophthalmol. 2002; 133: 78793.

12. Fuhrmeister H. A long-term study of morphological and functional developments after central serous chorioretinitis. Klin Monatsbal Augenheilkd. 1983; 182: 549-551.

13. Hayashi K, Hasegawa Y, Tokoro T. Indocyanine green angiography of central serous chorioretinopathy. Int Ophthalmol. 1986; 9: 37-41.

14. Prünte C. Indocyanine green angiography findings in central serous chorioretinopathy. Int Ophthalmol. 1995; 19: $77-82$.

15. Prünte $\mathbf{C}$, Flamme J. Choroidal capillary and venous congestion in central serous chorioretinopathy. Am J Ophthalmol. 1996; 121: 26-34.

16. Stanga PE, Lim JI, Hamilton P. Indocyanine green angiography in chorioretinal diseases: indications and interpretation: an evidence-based update. Ophthalmology, 2003; 110: 15-21.

17. Piccolino FC, Borgia L. Central serous chorioretinopathy and indocyanine green angiography. Retina, 1994; 14: 231-242.

18. Piccolino FC, Borgia L, Zinicola E, Zingirian M. Indocyanine green angiographic findings in central serous chorioretinopathy. Eye, 1995; 9: 324-332.
19. Seong HK, Bae JH, Kim ES, Han JR, Nam WH, Kim HK. Intravitreal Bevacizumab to Treat Acute Central Serous Chorioretinopathy: Short-Term Effect. Ophthalmologica, 2009; 223: 343-347.

Doi: $10.1159 / 000224782$.

20. Radhke N, Kalamkar C, Mukherjee A, Radhke S. Intravitreal Ziv-Aflibercept in Treatment of Naïve Chronic Central Serous Chorioretinopathy Related Choroidal Neovascular Membrane. Case Reports in Ophthalmol Med. 2017; 2017: 5036248.

21. Stewart MW, Rosenfeld PJ. Predicted biological activity of intravitreal VEGF Trap. Br J Ophthalmol. 2008; 92 (5): 667-668.

22. Stewart MW. Aflibercept (VEGF Trap-eye): the newest anti-VEGF. Br J Ophthalmol. 2012; 96 (9): 1157- 1158.

23. Stewart MW. Pharmacokinetics, pharmacodynamics and pre-clinical characteristics of ophthalmic drugs that bind VEGF. Expert Rev Clin Pharmacol. 2014; 7 (2): 167-180.

24. Kim $\mathbf{M}$, Lee $\mathbf{S}$, Lee $\mathbf{S}$. Intravitreal ranibizumab for acute central serous chorio-retinopathy. Ophthalmologica, 2013; 229: 152-157.

25. Jung BJ, Lee K, Park JH, Lee JH. Chorioretinal response to intravitreal Aflibercept injection in acute central serous chorioretinopathy. Int $\mathrm{J}$ Ophthalmol. 2019; 12 (12): 1865-1871.

\section{Authors' Designation and Contribution}

Muhammad Afzal Bodla; Professor: Concepts, Design, Manuscript editing, Manuscript Review.

Ali Afzal Bodla; Associate Professor: Data acquisition, Data analysis, Statistical Analysis, Manuscript Review.

Syeda Minahil Kazmi; Consultant Ophthalmologist: Literature Search Manuscript Preparation, Manuscript Editing.

Ayema Moazzam; Consultant Ophthalmologist: Statistical Analysis, Manuscript Preparation, Manuscript Editing. 